\title{
Tactile Sensing by the Sole of the Foot: Part I: Apparatus and Initial Experiments Toward Obtaining Dynamic Pressure Maps Useful for Stabilizing Standing, Walking, and Running of Humanoid Robots
}

\author{
Abhinav Kalamdani ${ }^{1}$, Chris Messom ${ }^{2}$, Mel Siegel ${ }^{1}$ \\ ${ }^{1}$ The Robotics Institute, Carnegie Mellon University, Pittsburgh, PA 15213, USA \\ ${ }^{2}$ IIMS, Massey University, Auckland, New Zealand.
}

\begin{abstract}
This paper introduces prototype experimental apparatus to investigate stability in standing, walking and running of humanoid robots using pressure sensing at the foot contact. The preliminary experiments show that the system can provide very good spatial or temporal resolution and these can be traded off each other given the problem at hand, such as the sparsely sampled whole foot during static balancing or the densely sampled impact point of the foot during walking or running. The prototype apparatus, experimental result, and dynamic models of the system will give insight into the nature of balance control.
\end{abstract}

Keywords - Tactile sensing, force/pressure sensing, humanoid robots

\section{INTRODUCTION}

We describe newly designed and constructed prototype apparatus, and initial experiments with it, that demonstrate its ability to deliver high spatial and temporal resolution tactilesensing data streams from the sole of a humanoid foot. Our eventual aim is to characterize and understand the role of tactile sensing by the human foot in stabilizing bipedal standing, walking, and running [1-3], and to apply this understanding to the corresponding problems in the design and control of the corresponding activities by humanoid robots [4-8]. Research has shown that human balance control is dependant on the force feedback provided by the pressure sensing in the human sole [1-2]. Understanding the characteristic of this system, including the dynamics of the feedback and control systems will provide significant insight into the nature of human and humanoid robot balance for standing, walking and running.

Our initial experiments demonstrate the fitness of the apparatus to generate, capture, and display high signal-tonoise ratio signals of the static pressure distribution under the realistically-loaded foot and - more important - to capture and display the relatively small changes in the pressure distribution that occur when an external destabilizing force applied at the knee, approximately perpendicular to the knee, in any azimuthal direction - causes the tibia to become offset a few degrees from its stable load-bearing pose.

\section{APPROACH}

Our prototype apparatus is a wooden frame, approximately one meter on each side, with a plywood base (Figure 1). Force-multiplying pulleys allow realistic loading of a prosthesis-like wooden leg-and-foot using easily-managed static weights (Figure 2). Although we have not considered using the apparatus in prosthesis evaluation it is likely that it will provide insight into design and evaluation of prosthetic feet.

The sensor per se (Figure 3) is a force sensitive resistor array sold by TekScan ${ }^{1}$ for medical diagnosis of foot problems. The sensors are inexpensive, but TekScan's monitoring hardware and software are both prohibitively expensive and generally ill-suited to the experimental scenarios we contemplate [9-16]. We therefore constructed our own monitor using analog current multiplexers (Figure 5) and a single board computer (SBC).

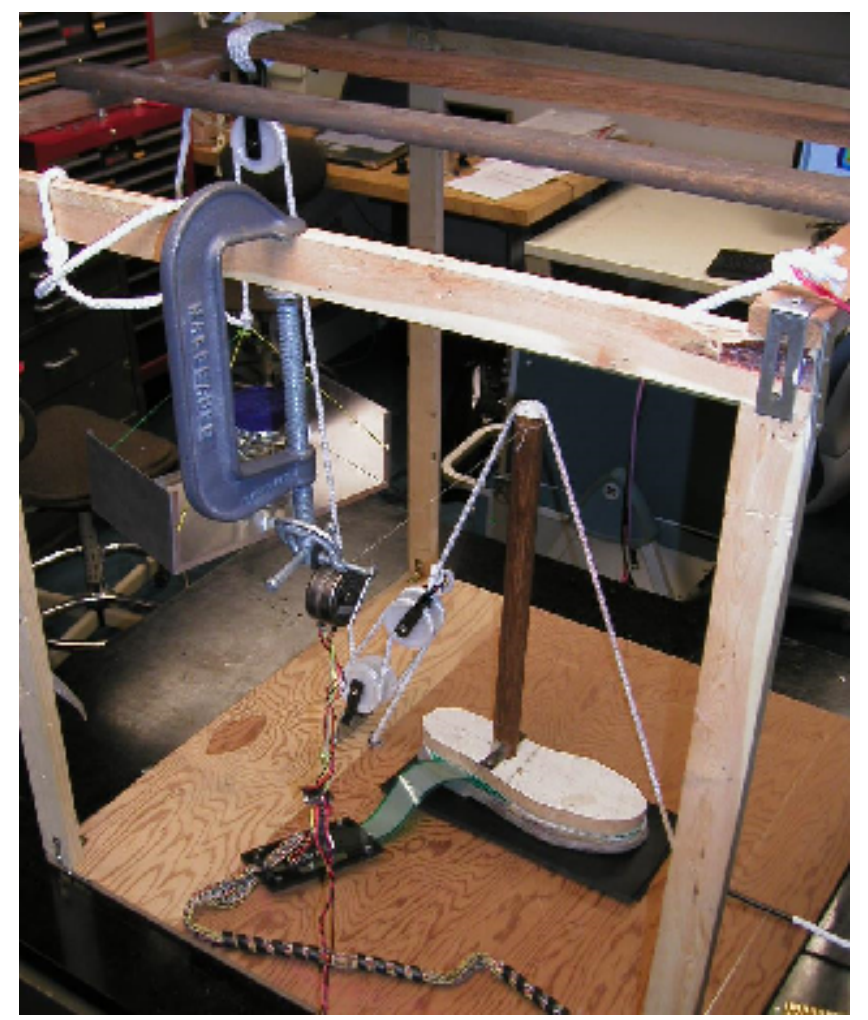

Figure 1. Overview of the mechanical apparatus.

\footnotetext{
${ }^{1}$ TekScan Corporation, South Boston MA, http://www.tekscan.com
} 


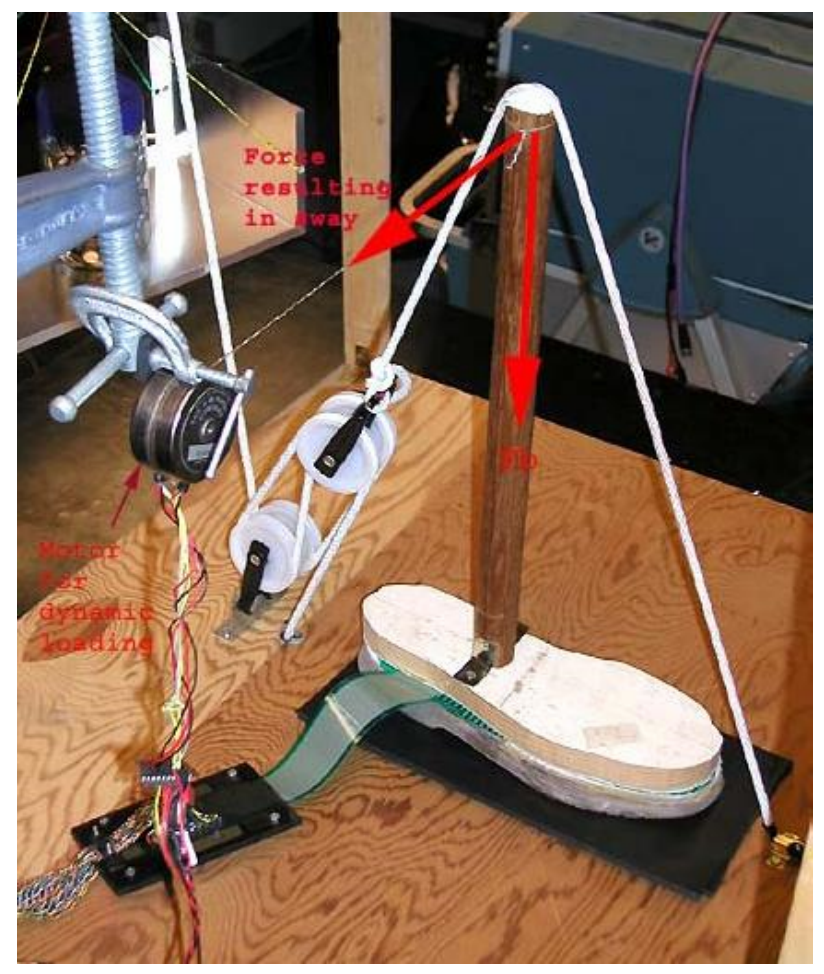

Figure 2. Detail of the foot, leg, static and destabilizing loading mechanisms.

On the SBC's low-level side it controls the multiplexers selecting the tactile element desired at each instant - and on its high-level side it communicates with a PC via a serial link. The PC generates the scan sequence that the controller will execute - single tactile element, full raster scan of all elements, low resolution raster scan of all elements, foveal pattern scanning, etc. - so as to trade off the available spatial and temporal resolutions, whose product is limited by the sampling rate of the controller's analog-to-digital controller. The PC also supports the user interface, including a falsecolor-mapped display of the recorded pressure maps.

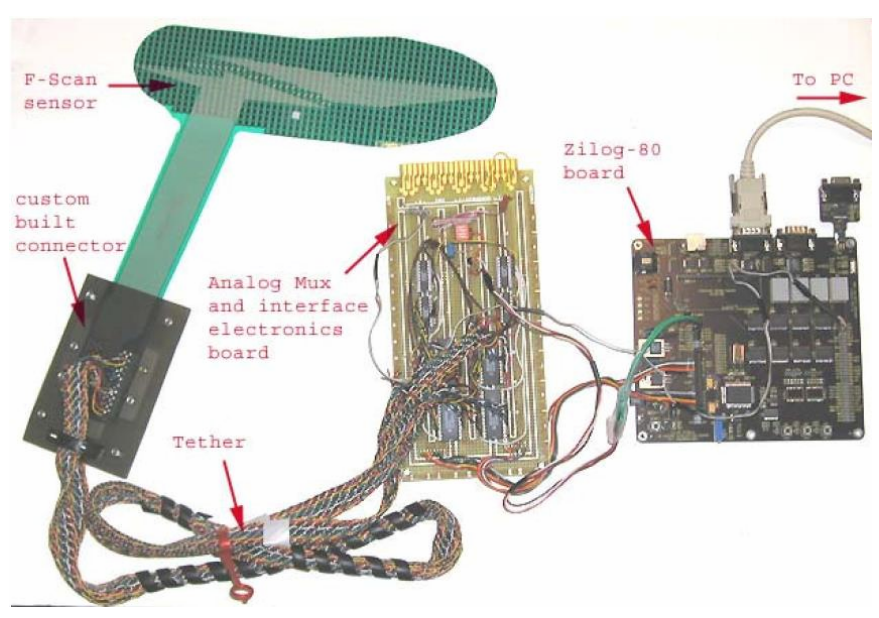

Figure 3. Sensor array, multiplexer, single-board controller.
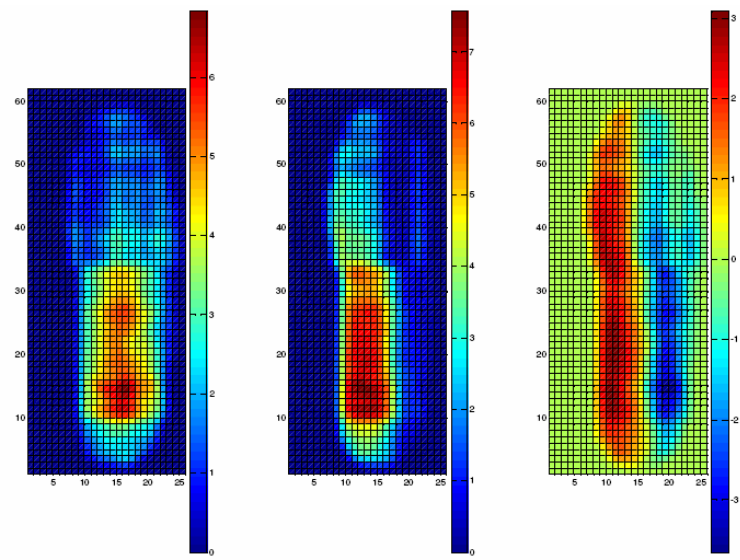

Figure 4. Pressure maps. Left map is stable standing. Middle is with a 3.6 degree leftward deflection of the knee. Right is the renormalized difference, clearly showing more pressure on left, less pressure on left, but relatively little front-back assymetry.

The disturbance (Figure 4) is currently generated by a stepper motor (Figure 2) - controlled by the PC's parallel port - that oscillates the tension in a string attached to the knee. The next step in our ongoing program will be to reverse the sign: to analyze the dynamical behavior of the pressure map so as to recognize and characterize disturbances, and generate control signals whereby, e.g., a pair of motors will restore stability.

A two-dimensional Gaussian smoothing filter of characteristic size a few tactile elements improves the interpretability of the display for the human eye-brain by eliminating high frequency noise due apparently to sensor manufacturing inconsistencies (Figure 6). How best to translate the dynamic pressure map into symbols and signals that can effect control actions to restore stability in response to disturbance is the topic next on our research agenda.

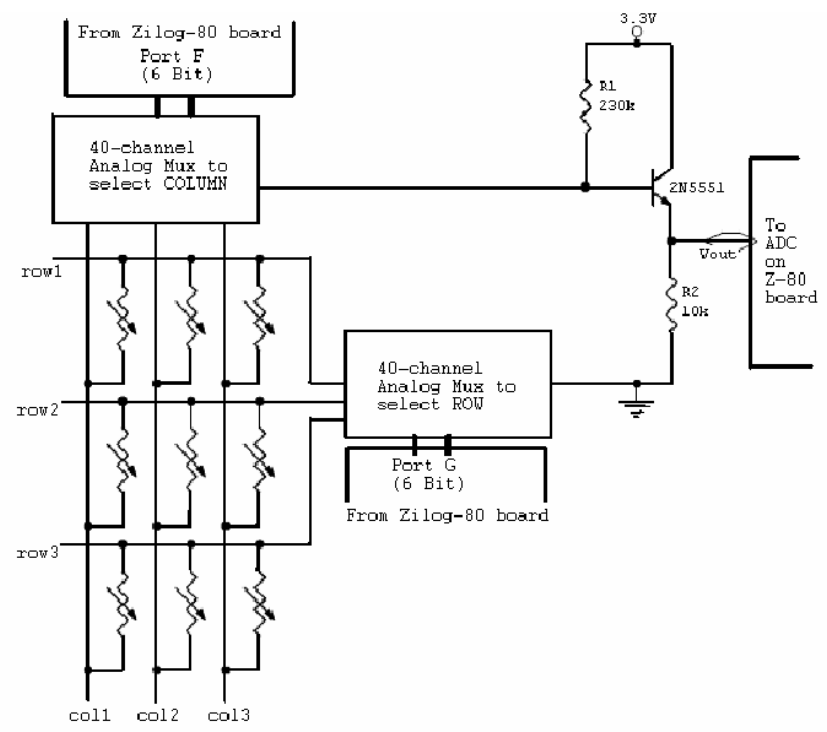

Figure 5. Sensor, analog multiplexers and interface circuit 


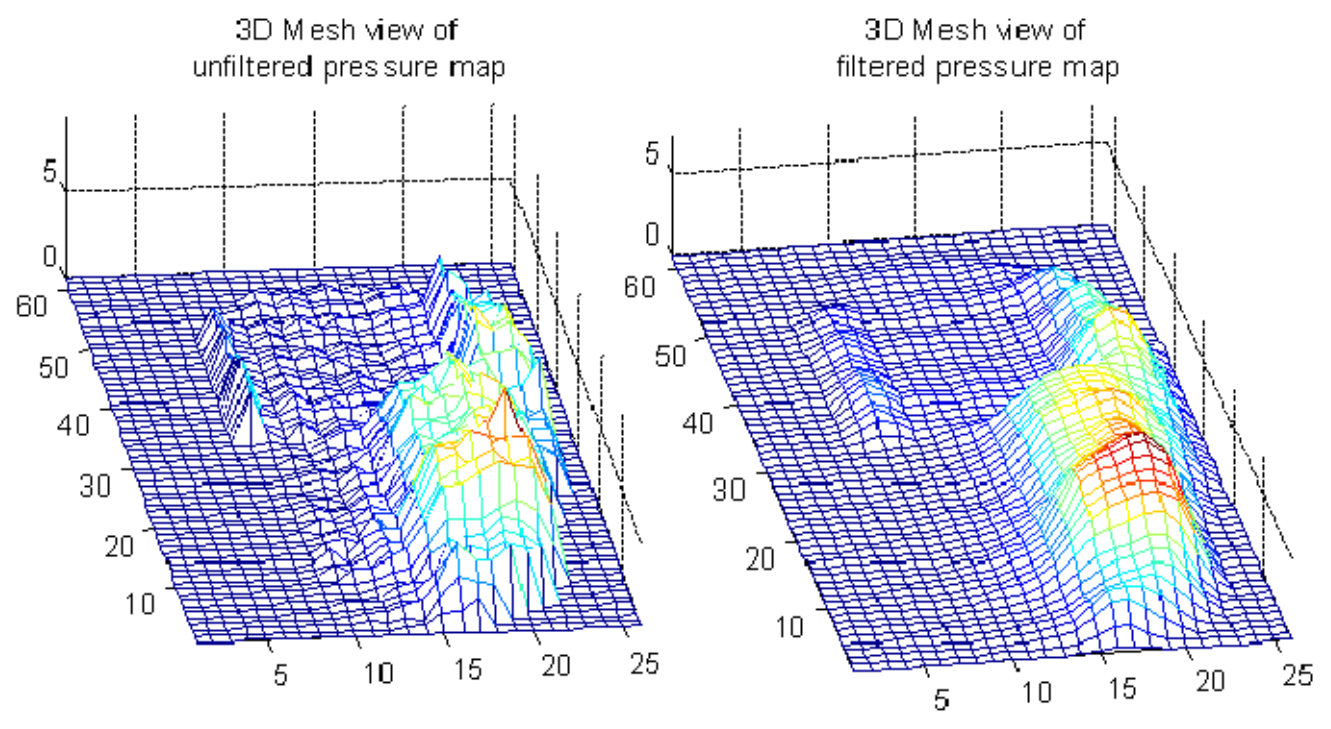

Figure 6. Filtered and unfiltered data

Table 1. Center-of-pressure, standard deviation, skew and peak pressure for right, left, anterior and posterior loading of the knee showing the initial conditions and relative changes to the initial conditions

\begin{tabular}{|c|c|c|c|c|c|c|c|c|c|c|}
\hline \multirow{2}{*}{$\begin{array}{l}\text { Parameters } \\
\text { (pixel units) }\end{array}$} & \multicolumn{2}{|c|}{ Initial Condition } & \multicolumn{2}{|c|}{$\begin{array}{l}\text { Right sway by } \\
3.6^{\circ}\end{array}$} & \multicolumn{2}{|c|}{$\begin{array}{l}\text { Left sway by } \\
3.6^{\circ}\end{array}$} & \multicolumn{2}{|c|}{$\begin{array}{l}\text { Anterior sway } \\
\text { by } 7.3^{\circ}\end{array}$} & \multicolumn{2}{|c|}{$\begin{array}{l}\text { Posterior sway } \\
\text { by } 6.0^{\circ}\end{array}$} \\
\hline & $\mathrm{X}$ axis & $\mathrm{Y}$ axis & $\mathrm{X}$ axis & $\mathrm{Y}$ axis & $\mathrm{X}$ axis & $\mathrm{Y}$ axis & $\mathrm{X}$ axis & $\mathrm{Y}$ axis & $\mathrm{X}$ axis & $\mathrm{Y}$ axis \\
\hline $\mathrm{COP}$ & 14.75 & 19.50 & 4.39 & 2.53 & -3.49 & 4.72 & -0.43 & 10.96 & 0.31 & -0.50 \\
\hline Std.dev. & 3.56 & 8.78 & -1.45 & 1.90 & -1.28 & 4.78 & -0.05 & 3.20 & 0.34 & 0.01 \\
\hline Skewness & 0.11 & 0.06 & -0.30 & 0.17 & 0.17 & 0.40 & 0.24 & 0.20 & -0.10 & -0.08 \\
\hline Peak Pres. & \multicolumn{2}{|c|}{$14.8 \mathrm{kPa}$} & \multicolumn{2}{|c|}{$20.6 \mathrm{kPa}$} & \multicolumn{2}{|c|}{$22.5 \mathrm{kPa}$} & \multicolumn{2}{|l|}{$16 \mathrm{kPa}$} & \multicolumn{2}{|c|}{$22.2 \mathrm{kPa}$} \\
\hline
\end{tabular}

Currently we are approaching the issue by computing the first few moments of the distribution - the center-of-pressure along and across the foot, and its width, and a measure of its asymmetry in these canonical directions. These six numbers - three measures times two directions - seem effectively and efficiently to capture the essence of the static and dynamic state of the system, whereas - at least at this early stage of the work - higher moments seem mostly just to fit the noise.

In future an image processing approach may be adopted for higher spatial frequency information in the signals, such as surface texture and surface unevenness. Identifying texture and other surface features may allow the control strategy to be adjusted for different surfaces as well as to identify situations where the foot is slipping or in other unstable configurations such as inadequate foot support contact geometry.

An additional challenge that is introduced, if image processing techniques are adopted, is that each "pixel" is sampled at a different point in time, and the scan patterns are in general not progressive, so additional signal processing techniques must be used to obtain an inferred time interpolated image before further processing. An advantage of a time interpolated image will be that there is no inherent image frame time at which the image is taken and so the inferred time interpolated image can be constructed for any given instant. The quality of the time interpolated image will not depend on the chosen time point, as sampling occurs continuously rather than in the periodic raster pattern of digital image capture systems.

\section{RESULTS}

Static lateral loading (Figure 2) of the tibia shows sensible changes in the pressure map (Figure 7), quantified as changes in the center-of-pressure, the width of the distribution (characterized by standard deviation), and the asymmetry of the distribution (characterized by skew). These measures are calculated using the first three moments of the pressure distribution along the width (x) and length (y) axes of the foot (Table 1).

Calibration with accuracy and precision that are adequate for the intended monitoring-and-control task is 


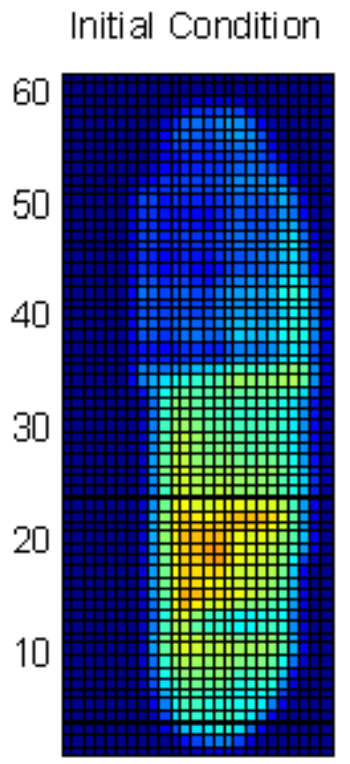

510152025

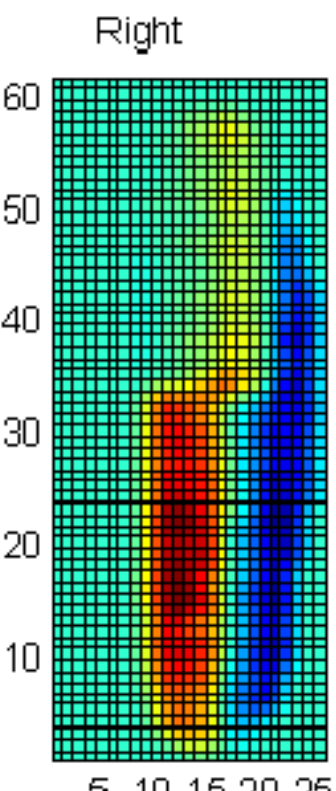

510152025
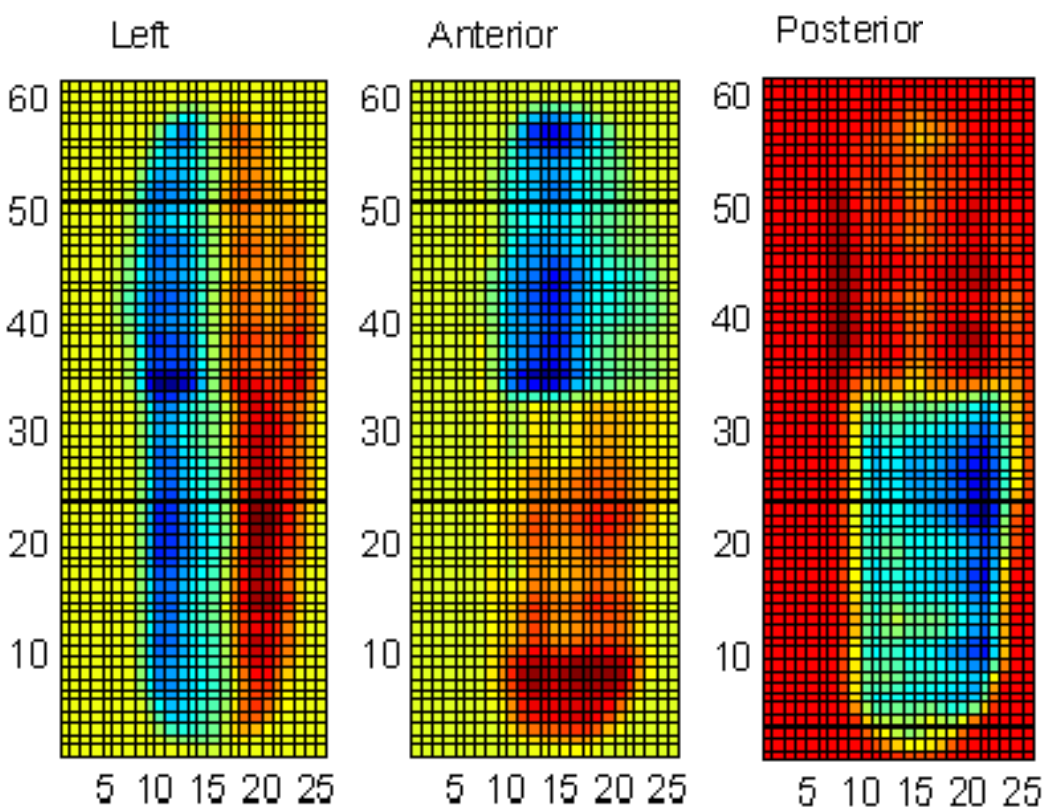

Figure 7. Difference map of right, left, anterior and posterior loading of the knee relative to the initial conditions

straightforward. The sum of the signals over all elements must equal the imposed load on the whole foot, so the known total weight and the known element spacing and count give the signal-to-pressure conversion factor. Measured shifts in pressure pattern - parameterized by offset-from-static angle and direction - left, right, front (anterior), and back (posterior) - are reported as change in center-of-pressure, width (standard deviation), and asymmetry (third moment, skew). These observables constitute a model for inversion of pattern change into tilt and direction, and from them magnitude and direction of the lateral perturbation, and from there finally into the opposing forces that the control system must apply to offset the perturbation.

\section{DISCUSSION}

Our foot tactile measurement system is able to provide good spatial and temporal resolution, with the relative tradeoff due to the limit of the single ADC. Our current design can be augmented to use two independent ADCs, doubling the bandwidth, but the tradeoff between spatial and temporal resolution will still exist. Our proof-of-principle system will have to be significantly modified to make use of more parallel sampling of the pressure sensor network. At this point the bandwidth between the PC and the system electronics becomes a bottleneck and would require use of faster communication protocols as well as compression to overcome this limitation. On the other hand, the apparatus is explicitly a prototype intended for pilot experiments: we know how to make it better next time, in large part because of what we learned from building and running this "quick and dirty" device.
As seen in Figure 4, 5, and 7, our current system has very high spatial resolution compared to the tactile regions of the human foot. Thus we expect that in practical running, walking, and standing control applications we will be able to reduce the spatial resolution - and correspondingly to increase the temporal resolution - enough to close the control loop substantially faster than the mechanical system's characteristic times.

The spatial and temporal resolution tradeoff will require different scanning policies for different modes of operation. During static balancing and standing the whole area of the foot must be sampled, but due to the temporal resolution required to maintain balance, a sparse sampling approach must be adopted. During walking and running the flight phase of the foot does not require dense spatial sampling but identifying the contact time of the foot collision with the ground requires high temporal resolution. This will require sparse spatial sampling around the heel strike region to identify when and where heal strike occurs and then transition to a denser spatial sampling as the heel is loaded so that a good measure of the heel strike pressure can be made. These sampling policies are an area of further research.

Quantitatively, currently a full raster scan of the system takes approximately $300 \mathrm{~ms}$, so even a sparse sampling that neglects only every other sensor node in both $\mathrm{x}$ - and $\mathrm{y}$-axes will result in a refresh rate in excess of $10 \mathrm{~Hz}$, well within the time constants of dynamic walking systems, although this refresh rate may need to be increased to match the performance and time constants of static balancing systems (standing in place vs. walking or running) where the neurodynamics and muscle time constants dominate rather than the time constants of the mechanical system. 
Additionally, documented problems with the commercial sensor per se include element-to-element sensitivity and linearity variation, hysteresis, and drift. The end-to-end calibration of the system in situ at the start of experiments overcomes most of these problems. Nevertheless, it is possible that subtle enough effects will come into play in natural running, walking, and standing that automatic dynamic recalibration will be required when experiments are extended to many hours, or perhaps just many minutes.

\section{REFERENCES}

[1] A. Kavounoudias, R. Roll, J.P. Roll, The plantar sole is a 'dynamometric map' for human balance control, Cognitive Neuroscience Neuroreport, 9(14), 1998, pp. 3247-3252.

[2] C. Maurer, T. Mergner, B. Bolha, F. Hlavacka, Human balance control during cutaneous stimulation of the plantar soles, Neurosci Lett., 302(1), 2001, pp. 45-8.

[3] D.C. Gravelle, C.A. Laughton, N.T. Dhruv, K.D. Katdare, J.B. Niemi, L.A. Lipsitz, J.J. Collins, Noise-enhanced balance control in older adults, Somatosensory Systems, Pain Neuroreport. 13(15), 2002, pp. 1853-1856.

[4] A.Goswami, Postural stability of biped robots and the foot-rotation indicator point, Int. Jour. of Rob. Res., vol 18, no.6, June 1999, pp. 523-533

[5] S.H.Collins, M.Wisse, A.Ruina, A three-dimensional passive-dynamic walking robot with two legs and knees, Int. Jour. Rob. Res. vol.20, no.7, July 2001, pp.607-615
[6] P.Sardain, G.Bessonnet, Forces acting on a biped robot, center of pressure-zero moment point, IEEE Tran. on Sys. Man \& Cyb. - Part A: Sys. \& Humans, vol.34, no.5, sept 2004, pp.630-637

[7] P.Sardain, G.Bessonnet, Gait analysis of a human walker wearing robot feet as shoes, Proc. of 2001 IEEE Int. Conf. on Rob. \& Auto., May 2001, p 2285-2292

[8] T.Tanaka, H.Takeda, T.Izumi, S.Ino, T.Ifukube, Effects on the location of the center of gravity and the foot pressure contribution to standing balance associated with ageing, Ergonomics, 1999, vol.42, no.7, 9971010

[9] F.Vecchi, C.Freschi, S.Micera, A.M.Sabatini, P.Dario, R.Sacchetti, Experiemental evaluation of two commercial force sensors for applications in biomechanics and motor control, 5th Ann. Conf. of Int. FES 2000.

[10] H.Zhu, N.Maalej, J.G.Webster, W.J.Tompkins, P.B.Y.Rita, J.J.Wertsch, An umbilical data-acquisition system for measuring pressures between the foot and shoe, IEEE Trans. on Biomed. Engg. vol.37. no.9, sept 1990

[11] S.J.Morris, A shoe-integrated sensor system for wireless gait analysis and real-time therapeutic feedback, a doctor of science thesis at MIT, June 2004.

[12] Z.P.Luo, L.J.Berglund, K.N.An, Validation of F-Scan pressure sensor system: A technical note, Jour. of Rehab. Res and Dev. vol.35, no.2, june 1998, pp.186-191

[13] T.Sumiya, Y.Suzuki, T.Kasahara, H.Ogata, Sensing stability and dynamic response of the F-Scan in-shoe sensing system: A technical note, Jour. of Rehab. Res. \& Dev., Vol. 35 No.2, June 1998, p 192-200

[14] J.Woodburn, P.S.Helliwell, Observations on the F-Scan in-shoe pressure measuring system, Clin. Biomech. vol. 12, No.3, 1997, p S16 
[15] E.Morin, S.Reid, J.M.Eklund, H.Lay, Y.Lu, J.Stevenson, J.T.Bryant, Comparison of ground reaction forces measured with a force plate, FScan and multiple individual force sensors, Queen's Univ., Ontario, Canada.

[16] M.Lowe, A.King, E.Lovett, T.Papakostas, Flexible tactile sensor technology: bringing haptics to life, Sensor review, vol 24, no.1, 2004, pp.33-36 